\title{
Analysis of BTO-on-Si-waveguides for energy-efficient electro-optical modulators
}

Steglich, Patrick, Mai, Andreas

Patrick Steglich, Andreas Mai, "Analysis of BTO-on-Si-waveguides for energyefficient electro-optical modulators," Proc. SPIE 11775, Integrated Optics:

Design, Devices, Systems and Applications VI, 117750L (18 April 2021); doi: $10.1117 / 12.2592501$

SPIE. Event: SPIE Optics + Optoelectronics, 2021, Online Only 


\title{
Analysis of BTO-on-Si-waveguides for energy-efficient electro-optical modulators
}

\author{
Patrick Steglich ${ }^{\mathrm{a}, \mathrm{b}}$ and Andreas Mai ${ }^{\mathrm{a}, \mathrm{b}}$ \\ aHP - Leibniz-Institut für innovative Mikroelektronik, 15236 Frankfurt (Oder), Germany \\ ${ }^{\mathrm{b}}$ Technische Hochschule Wildau, 15745 Wildau, Germany
}

\begin{abstract}
In this work, we analyze a horizontal slot waveguide configuration, which evaluates the potential for an integration of barium titanate (BTO) based modulators into a photonic integrated circuit (PIC) technology based on siliconon-insulator (SOI) wafer. The waveguide configuration consists of a doped crystal silicon layer, a $\mathrm{Ba}_{0.7} \mathrm{Sr}_{0.3} \mathrm{TiO}_{3}$ (BST) template layer, a barium titanate (BTO) layer and a doped poly-silicon layer on top. In contrast to current approaches, we analyze the performance of this waveguide-structure by using a vertical electrode configuration that is formed by the doped silicon layer. In this way, the electric field strength is dramatically increased compared to current horizontal electrode configuration.
\end{abstract}

Keywords: Pockels effect, Ferroelectric Materials, Barium Titanate, Electro-Optical Modulators, Slot Waveguide, Pockels Modulator

\section{INTRODUCTION}

Silicon photonics has become the major platform for optical communication systems due to its high scalability and mass-production capability. ${ }^{1}$ Several electro-optical effects like the Pockels, ${ }^{2}$ DC Kerr ${ }^{3,4}$ or electric fieldinduced Pockels effect ${ }^{5}$ can be used for optical modulators. However, coherent optical communication ${ }^{6}$ requires a highly linear dependence of the modulated optical signal from the electrical input signal, while the employed electro-optical effect should be off-resonant in order to modulate the refractive index but not the absorption. In this way, higher order modulation formats like m-th quadrature amplitude modulation (QAM) can be realized.

Unfortunately, silicon lacks intrinsically from an efficient Pockels effect, also known as the linear electrooptical effect (LEOE), ${ }^{7}$ making high-speed modulation with advanced modulation formats challenging, while the energy-consumption is relatively high and the bandwidth is limited. As a consequence, heterogeneous integration of novel materials like electro-optical polymers, ${ }^{8-11}$ ferroelectric materials ${ }^{12,13}$ or III-V materials is required. ${ }^{14}$ One focus of current research lies on the hybrid-integration of ferroelectric materials like barium titanate (BTO) into silicon-based photonic integrated circuits (PIC), ${ }^{15}$ giving rise to an extraordinary Pockels effect. ${ }^{16-18}$

We present an analysis of a horizontal slot waveguide configuration consisting of barium titanate (BTO) on silicon using a $\mathrm{Ba}_{0.7} \mathrm{Sr}_{0.3} \mathrm{TiO}_{3}$ (BST) layer as template material. We propose the use of a vertical electrode configuration, which is formed by the doped silicon layer. Using BTO in the slot waveguide leads to a large field confinement inside the BTO layer. The BTO layer is out-of-plane oriented. This allows to provide a larger Pockels coefficient and results in a large LEOE in the center layer. State-of-the-art waveguides use bulk metal electrodes beside the waveguide in order to generate a horizontal electric field. This, however, is accompanied with a relatively large electrode distance and, hence, this makes it challenging to enable a sufficient co-integration with state-of-the-art electronic technologies.

In contrast to current approaches, we analyze the performance of this waveguide-structure by using a vertical electrode configuration that is formed by doped silicon layer. In this way, the electric field strength is dramatically increased compared to current horizontal electrode configurations. This allows the efficient use of the Pockels effect, which results in lower driver voltages.

Further author information (Patrick Steglich):

steglich@ihp-microelectronics.com

Integrated Optics: Design, Devices, Systems and Applications VI, edited by Pavel Cheben, Jiří Čtyroký,

Iñigo Molina-Fernández, Proc. of SPIE Vol. 11775, 117750L · (C) 2021 SPIE

CCC code: $0277-786 \mathrm{X} / 21 / \$ 21 \cdot$ doi: $10.1117 / 12.2592501$

Proc. of SPIE Vol. 11775 117750L-1 


\section{RESULTS}

The most common electrode configuration is shown in Figure 1a. In this case, the distance between two electrodes is about $2 \mu \mathrm{m}$ and a strontium titanate (STO) template layer is used to get $a$-axis BTO. ${ }^{2}$ In this work, we propose a vertical electrode configuration, as shown in Figure 1b. This has the advantage of a lower electrode distance. However, since the external electric field is then aligned along the $z$-direction, the Pockels effect is dramatically reduced. ${ }^{19}$ Therefore, it is necessary to grow $c$-axis BTO, i.e. the $c$-axis lies perpendicular to the plane of the substrate. This can be achieved by using a solid solution of barium and strontium titanate $\left(\mathrm{Ba}_{0.7} \mathrm{Sr}_{0.3} \mathrm{TiO}_{3}\right.$, BST). For example, a $c$-axis oriented epitaxial BTO film has been grown on (001) Si by reactive molecular-beam epitaxy. ${ }^{20}$

In the following, we use $c-S i=220 \mathrm{~nm}, B S T=30 \mathrm{~nm}, B T O=80 \mathrm{~nm}$ and $a-S i=80 \mathrm{~nm}$ as layer thickness, which are typical values for PIC technologies and results in an electrode distance of $110 \mathrm{~nm}$. In fact, a reduced electrode distance is lowering the required driving voltage of electro-optical modulators based on the Pockels effect, which motivates our investigation on a vertical electrode configuration.
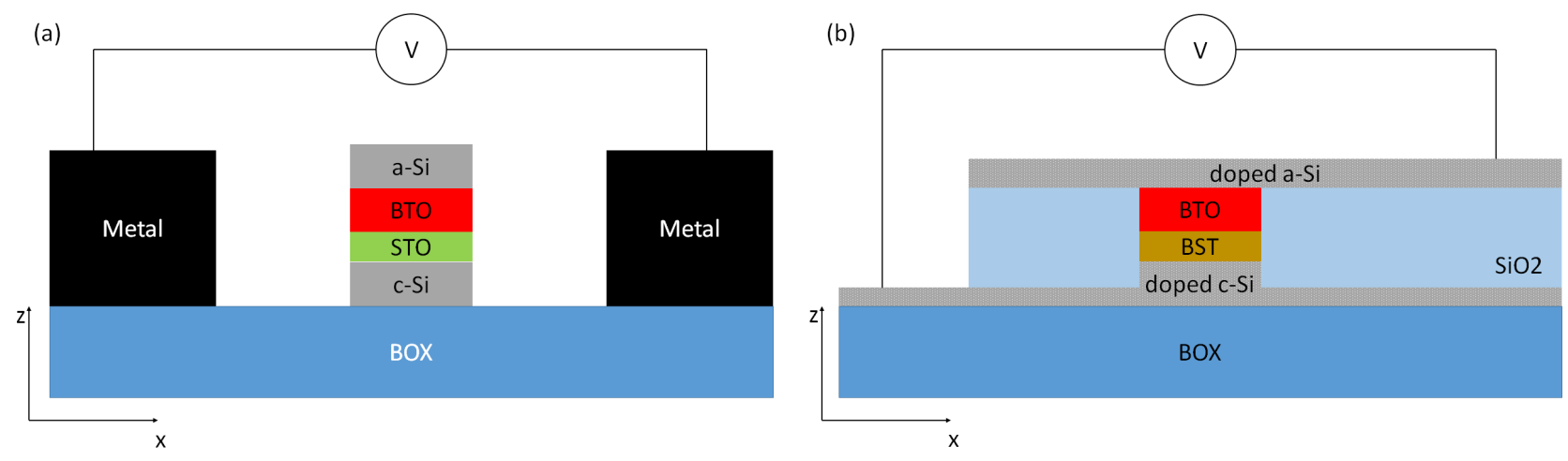

Figure 1. (a) Typical electrode configuration for an a-axis BTO, using a STO template layer. (b) A vertical electrode configuration is proposed. A BST template layer is used to get $c$-axis BTO.

The Pockels effect describes the electric field induced refractive index change described by the change of an ordinary $\left(\Delta n_{o}\right)$ and extraordinary $\left(\Delta n_{e}\right)$ refractive index. The optical indicatrix

$$
a_{11} x^{2}+a_{22} y^{2}+a_{33} z^{2}+2 a_{23} y z+2 a_{31} z x+2 a_{12} y x=1
$$

is related to the Pockels coefficients $r_{i} j$ of BTO using the tensorial expression

$$
\left[\begin{array}{c}
a_{11}-\frac{1}{n_{1}} \\
a_{22}-\frac{1}{n_{2}} \\
a_{33}-\frac{1}{n_{3}} \\
a_{23} \\
a_{31} \\
a_{12}
\end{array}\right]=\left[\begin{array}{ccc}
0 & 0 & r_{13} \\
0 & 0 & r_{23} \\
0 & 0 & r_{33} \\
0 & r_{42} & 0 \\
r_{51} & 0 & 0 \\
0 & 0 & 0
\end{array}\right]\left[\begin{array}{c}
E_{x} \\
E_{y} \\
E_{z}
\end{array}\right],
$$

where $E_{x}, E_{x}$ and $E_{x}$ represent the external electric field in $x, y$ and $z$-direction, respectively. Here, we assume a BTO film mostly with c-axis ferroelectric domain orientation, as it is typically the case. ${ }^{19,21}$ The Pockels coefficient is described by $r_{i j}$. Here, we used the in the Voigt notation (i.e. $x=1, y=2, z=3, x x=1, y y=2$, $z z=3, y z=4, x z=5, x y=6)$. The zero components in Eq. 2 can be explained by the crystallographic point group $4 \mathrm{~mm}$ of BTO. This also leads us to the relations $r_{13}=r_{23}$ and $r_{42}=r_{51}$. The Pockels coefficients for stress-free BTO are $r_{13}=r_{23}=10.2 \pm 0.6 \mathrm{pm} / \mathrm{V}, r_{42}=r_{51}=1300 \pm 100 \mathrm{pm} / \mathrm{V}$ and $r_{33}=105 \pm 10 \mathrm{pm} / \mathrm{V}$, as reported by Zgonik et al. ${ }^{22}$

Presuming a small change of the refractive index and restricting the external electric field to be aligned along the $z$-direction $\left(E=E_{z}\right)$ and taking into account that $n_{1}=n_{2}=n_{o}$ and $n_{3}=n_{e}$, the indicatrix (Eq. 2) can be 
(a)

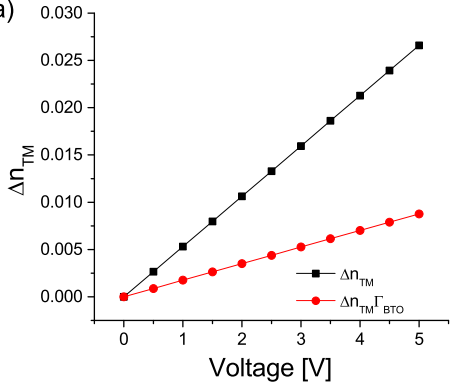

(b)

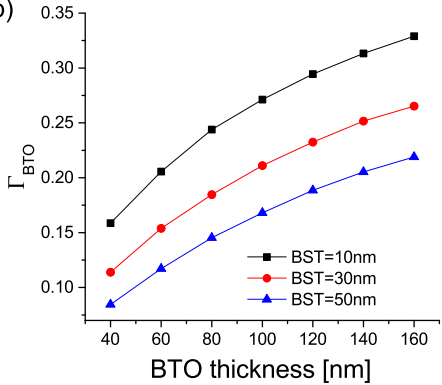

(c)

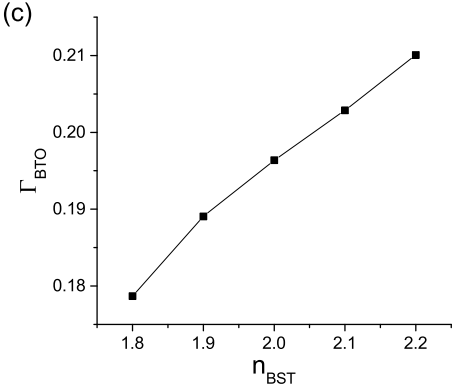

Figure 2. (a) Refractive index change as a function of the external voltage. The upper function (black squares) shows the refractive index change of the TM-mode and the lower function (red dots) shows the same function but multiplied with the field confinement factor (calculated for $B S T=30 \mathrm{~nm}, \mathrm{BTO}=120 \mathrm{~nm}$ ). (b) Calculated field confinement factor as function of BTO and BST thickness. (c) Field confinement factor as function of BSTs refractive index.

approximated as

$$
\frac{x^{2}}{\left(n_{o}-\frac{1}{2} n_{o}^{3} r_{13} E_{z}\right)^{2}}+\frac{z^{2}}{\left(n_{e}-\frac{1}{2} n_{e}^{3} r_{33} E_{z}\right)^{2}}=1
$$

Here, we have neglected the $y$-component because the the light can only be guided as TE-mode (electric field along $x$-direction) or TM-mode (electric field along $z$-direction).

In case of TE-mode, the refractive index change can be described by

$$
\Delta n_{T E}=-\frac{1}{2} n_{o}^{3} r_{13} \frac{V}{d}
$$

and in if the guided mode is TM, the refractive index change is given by

$$
\Delta n_{T M}=-\frac{1}{2} n_{e}^{3} r_{33} \frac{V}{d}
$$

where $V / d$ equals $E_{z}$ and $V$ and $d$ are the external voltage and electrode distance, respectively. The ordinary and extraordinary refractive index is $n_{o}=2.3$ and $n_{e}=2.27$, respectively. ${ }^{23}$ For our simulation study, we use the same refractive index for $c-S i$ and $a-S i$ of $3.47 .{ }^{24}$ The refractive index of BST is $1.86 .{ }^{25}$

From Eq. 4 and Eq. 5 it becomes apparent that the TM-mode is preferable because it allows the use of the Pockels coefficient $r_{33}$, which is ten times larger than $r_{13}$ and, therefore, allows the efficient use of the proposed vertical device concept. Please note that we use a Pockels coefficient of $r_{33}=105 \pm 10 \mathrm{pm} / \mathrm{V}$ for our study, as reported by Zgonik et al. ${ }^{22}$ Abel et al. have reported a Pockels coefficient of $r_{33}=342 \pm 93 \mathrm{pm} / \mathrm{V}$ in thin film BTO. ${ }^{2}$ This value is ten times higher than that of lithium niobate $\left(r_{33}=30 \mathrm{pm} / \mathrm{V}^{26}\right)$. In the following we will use the lower value of $r_{33}=105 \pm 10 \mathrm{pm} / \mathrm{V}$.

Figure 2 shows the simulation results. The values are calculated for a wavelength of $1550 \mathrm{~nm}$ and a refractive index for BST of 2.0. The refractive index change as a function of the external voltage is depicted in Figure 2a. The upper function (black squares) shows the refractive index change of the TM-mode and the lower function (red dots) shows the same function but multiplied with the field confinement factor (calculated for BST=30 nm, $\mathrm{BTO}=120 \mathrm{~nm}$ ). The optical field confinement is defined as the ration of the optical power inside the BTO layer and the entire optical power

$$
\Gamma_{B T O}=\frac{P_{B T O}}{P_{\infty}},
$$

where the optical power is calculated by

$$
P=\iint \frac{1}{2} \operatorname{Re}\left\{\mathbf{E} \times \mathbf{H}^{*}\right\} \mathrm{d} x \mathrm{~d} y,
$$


where $E$ and $H$ are the electric and magnetic field vectors, respectively. ${ }^{27-29}$

Figure $2 \mathrm{~b}$ shows the calculated field confinement factor as function of BTO and BST thickness. From this fugure it is appearend that the optical field confinement inside the BTO layer is increased by increasing the BTO thickness, while the BST thickness needs to be minimized. Since the refractive index of BST can vary and depends strongly on the deposition method and conditions, we have calculated the influence of the BSTs refractive index on the optical field confinement inside the BTO layer, as shown in Figure 2c. It is revealed that a high refractive index of BST is increasing the field confinement and therefore the overall refractive index change due to the Pockels effect.

\section{CONCLUSIONS}

We have analyzed a vertical electrode configuration by employing $c$-axis BTO. It is revealed that the Pockels coefficient $r_{33}$ can be efficiently used by designing a horizontal slot waveguide. A BTO thickness of $120 \mathrm{~nm}$ is recommended, while the BST thickness should be minimized and a high refractive index of BST is preferable. From our results it can be concluded that an energy-efficient Pockels modulator can be realized from the proposed waveguide configuration, while it provides perspectives towards BTO-based photonic devices that are hybridintegrated into a silicon-based PIC technology.

\section{ACKNOWLEDGMENTS}

This work was supported in part by the European Regional Development Fund under Grant 10.13039/501100008530.

\section{REFERENCES}

[1] Rahim, A., Goyvaerts, J., Szelag, B., Fedeli, J., Absil, P., Aalto, T., Harjanne, M., Littlejohns, C., Reed, G., Winzer, G., Lischke, S., Zimmermann, L., Knoll, D., Geuzebroek, D., Leinse, A., Geiselmann, M., Zervas, M., Jans, H., Stassen, A., Domínguez, C., Muñoz, P., Domenech, D., Giesecke, A. L., Lemme, M. C., and Baets, R., "Open-access silicon photonics platforms in europe," IEEE Journal of Selected Topics in Quantum Electronics 25(5), 1-18 (2019).

[2] Abel, S., Eltes, F., Ortmann, J. E., Messner, A., Castera, P., Wagner, T., Urbonas, D., Rosa, A., Gutierrez, A. M., Tulli, D., et al., "Large pockels effect in micro-and nanostructured barium titanate integrated on silicon," Nature materials 18(1), 42-47 (2019).

[3] Steglich, P., Mai, C., Villringer, C., Pulwer, S., Casalboni, M., Schrader, S., and Mai, A., "Quadratic electro-optic effect in silicon-organic hybrid slot-waveguides," Optics letters 43(15), 3598-3601 (2018).

[4] Steglich, P., Villringer, C., Dietzel, B., Mai, C., Schrader, S., Casalboni, M., and Mai, A., "On-chip dispersion measurement of the quadratic electro-optic effect in nonlinear optical polymers using a photonic integrated circuit technology," IEEE Photonics Journal 11(3) (2019).

[5] Steglich, P., Villringer, C., Dietzel, B., Mai, C., Schrader, S., Casalboni, M., and Mai, A., "Electric fieldinduced linear electro-optic effect observed in silicon-organic hybrid ring resonator," IEEE Photonics Technology Letters 32(9), 526-529 (2020).

[6] Kikuchi, K., "Fundamentals of coherent optical fiber communications," Journal of Lightwave Technology 34(1), 157-179 (2016).

[7] Steglich, P., Mai, C., Villringer, C., and Mai, A., "Direct observation and simultaneous use of linear and quadratic electro-optical effects," Journal of Physics D: Applied Physics 53(12), 125106 (2020).

[8] Steglich, P., Mai, C., Villringer, C., Dietzel, B., Bondarenko, S., Ksianzou, V., Villasmunta, F., Zesch, C., Pulwer, S., Burger, M., Bauer, J., Heinrich, F., Schrader, S., Vitale, F., Matteis, F. D., Prosposito, P., Casalboni, M., and Mai, A., "Silicon-organic hybrid photonics: Overview of recent advances, electro-optical effects and cmos-integration concepts," Journal of Physics: Photonics (2021).

[9] Steglich, P., Mai, C., and Mai, A., "Silicon-organic hybrid photonic devices in a photonic integrated circuit technology," ECS Journal of Solid State Science and Technology 8(11), Q217-Q221 (2019). 
[10] Steglich, P., Mai, C., Bondarenko, S., Villringer, C., Pulwer, S., Zesch, C., Dietzel, B., Schrader, S., Vitale, F., de Matteis, F., Casalboni, M., and Mai, A., "Functionalized materials for integrated photonics: Hybrid integration of organic materials in silicon- based photonic integrated circuits for advanced optical modulators and light-sources," in [2019 PhotonIcs Electromagnetics Research Symposium - Spring (PIERS-Spring)], 3019-3027 (2019).

[11] Steglich, P., Mai, C., Stolarek, D., Lischke, S., Kupijai, S., Villringer, C., Pulwer, S., Heinrich, F., Bauer, J., Meister, S., et al., "Partially slotted silicon ring resonator covered with electro-optical polymer," in [Silicon Photonics and Photonic Integrated Circuits V], 9891, 98910R, International Society for Optics and Photonics (2016).

[12] Xu, M., He, M., Zhang, H., Jian, J., Pan, Y., Liu, X., Chen, L., Meng, X., Chen, H., Li, Z., et al., "High-performance coherent optical modulators based on thin-film lithium niobate platform," Nature communications 11(1), 1-7 (2020).

[13] Eltes, F., Mai, C., Caimi, D., Kroh, M., Popoff, Y., Winzer, G., Petousi, D., Lischke, S., Ortmann, J. E., Czornomaz, L., Zimmermann, L., Fompeyrine, J., and Abel, S., "A batio3-based electro-optic pockels modulator monolithically integrated on an advanced silicon photonics platform," Journal of Lightwave Technology 37(5), 1456-1462 (2019).

[14] Pintus, P., Zhang, Z., Pinna, S., Tran, M. A., Jain, A., Kennedy, M., Ranzani, L., Soltani, M., and Bowers, J. E., "Characterization of heterogeneous inp-on-si optical modulators operating between $77 \mathrm{k}$ and room temperature," APL Photonics 4(10), 100805 (2019).

[15] Mai, A., Steglich, P., Mai, C., Simon, S., and Scholz, R., "Electronic-photonic wafer-level technologies for fast prototyping and application specific solutions," in [2019 PhotonIcs Electromagnetics Research Symposium - Spring (PIERS-Spring)], 249-255 (2019).

[16] Eltes, F., Ortmann, J. E., Castera, P., Urbonas, D., Caimi, D., Czornomaz, L., Sanchis, P., Fompeyrine, J., and Abel, S., "Silicon-integrated high-speed modulators based on barium titanate with record-large pockels coefficients," in [2019 Conference on Lasers and Electro-Optics Europe European Quantum Electronics Conference (CLEO/Europe-EQEC)], 1-1 (2019).

[17] Eltes, F., Fompeyrine, J., and Abel, S., "Ultra-efficient optical switching based on a large pockels effect embedded in silicon photonics," in [2020 Optical Fiber Communications Conference and Exhibition (OFC)], $1-3(2020)$.

[18] Eltes, F., Fompeyrine, J., and Abel, S., "A strong pockels effect in integrated photonic circuits," in [2020 IEEE Photonics Conference (IPC)], 1-1 (2020).

[19] Messner, A., Eltes, F., Ma, P., Abel, S., Baeuerle, B., Josten, A., Heni, W., Caimi, D., Fompeyrine, J., and Leuthold, J., "Plasmonic ferroelectric modulators," J. Lightwave Technol. 37, 281-290 (Jan 2019).

[20] Vaithyanathan, V., Lettieri, J., Tian, W., Sharan, A., Vasudevarao, A., Li, Y. L., Kochhar, A., Ma, H., Levy, J., Zschack, P., Woicik, J. C., Chen, L. Q., Gopalan, V., and Schlom, D. G., "c-axis oriented epitaxial batio3 films on (001) si," Journal of Applied Physics 100(2), 024108 (2006).

[21] Rosa, A., Tulli, D., Castera, P., Gutierrez, A. M., Griol, A., Baquero, M., Vilquin, B., Eltes, F., Abel, S., Fompeyrine, J., and Sanchis, P., "Barium titanate (batio3) rf characterization for application in electro-optic modulators," Opt. Mater. Express 7, 4328-4336 (Dec 2017).

[22] Zgonik, M., Bernasconi, P., Duelli, M., Schlesser, R., Günter, P., Garrett, M., Rytz, D., Zhu, Y., and Wu, X., "Dielectric, elastic, piezoelectric, electro-optic, and elasto-optic tensors of batio 3 crystals," Physical review B 50(9), 5941 (1994).

[23] Abel, S., Caimi, D., Sousa, M., Stöferle, T., Rossel, C., Marchiori, C., Chelnokov, A., and Fompeyrine, J., "Electro-optical properties of barium titanate films epitaxially grown on silicon," in [Oxide-based Materials and Devices III], 8263, 82630Y, International Society for Optics and Photonics (2012).

[24] Salzberg, C. D. and Villa, J. J., "Infrared refractive indexes of silicon germanium and modified selenium glass*," J. Opt. Soc. Am. 47, 244-246 (Mar 1957).

[25] Li, S., Yang, Y., Liu, L., Liu, W., and Wang, S., "The preparation and refractive index of bst thin films," Physica B: Condensed Matter 403(17), 2618-2623 (2008).

[26] Rabiei, P., Ma, J., Khan, S., Chiles, J., and Fathpour, S., "Heterogeneous lithium niobate photonics on silicon substrates," Opt. Express 21, 25573-25581 (Oct 2013). 
[27] Steglich, P., "Silicon-on-insulator slot waveguides: Theory and applications in electro-optics and optical sensing," in [Emerging Waveguide Technology], 187-210, IntechOpen (2018).

[28] Steglich, P., Villringer, C., Dümecke, S., Michel, Y. P., Casalboni, M., and Schrader, S., "Silicon-oninsulator slot-waveguide design trade-offs," in [2015 International Conference on Photonics, Optics and Laser Technology (PHOTOPTICS)], 2, 47-52, IEEE (2015).

[29] Steglich, P., Villringer, C., Pulwer, S., Casalboni, M., and Schrader, S., "Design optimization of siliconon-insulator slot-waveguides for electro-optical modulators and biosensors," in [Photoptics 2015], 173-187, Springer (2016). 